\title{
Infrared Hide-and-Seek: Vibrational Excitons Conceal Surfactants at the Air/Water Interface
}

\author{
Kimberly A. Carter-Fenk ${ }^{\dagger}$, Kevin Carter-Fenk ${ }^{\dagger}$, Michelle E. Fiamingo, Heather C. Allen*, and John M. Herbert* \\ Department of Chemistry and Biochemistry, The Ohio State University, Columbus, Ohio 43210, United States
}

\begin{abstract}
Surface-sensitive vibrational spectroscopy is a common tool for measuring molecular organization and intermolecular interactions at interfaces. Peak intensity ratios are typically used to extract molecular information from one-dimensional spectra but vibrational coupling between surfactant molecules can manifest as signal depletion in one-dimensional spectra. Through a combination of experiment and theory, we demonstrate the emergence of vibrational excitons in infrared reflectionabsorption spectra of soluble and insoluble surfactants at the air/water interface. Vibrational coupling yields a significant decrease in peak intensities corresponding to $\mathrm{C}-\mathrm{F}$ vibrational modes of perfluorooctanoic acid molecules. Vibrational excitons also form between arachidic acid surfactants within a compressed monolayer, manifesting as signal reduction of $\mathrm{C}-\mathrm{H}$ stretching modes. The aqueous phase ionic composition impacts surfactant intermolecular distances, thereby modulating vibrational coupling strength between surfactants. Our results serve as a cautionary tale against employing alkyl and fluoroalkyl vibrational peak intensities in analyses that are ubiquitous in interface science.
\end{abstract}

\section{Introduction}

Elucidating structure at the air/water interface is important to understanding chemical reactivity in biological, environmental, and industrial systems. Surfactants comprise an abundant class of amphiphilic molecules that partition at the air/water interface with vibrational modes that are typically well-separated from those of water, offering a sensitive probe of interfacial adsorption and organization via vibrational spectroscopy. ${ }^{1}$ Moreover, surfactant molecules spontaneously aggregate into micelles at high concentrations, ${ }^{2}$ and strong vibrational couplings play a central role in the spectra of self-aggregating nanostructures. ${ }^{3}$ Vibrational excitons emerge from the coupling of quasi-degenerate vibrational states and have the effect of depleting local signal intensity in one-dimensional (1D) spectroscopic measurements. ${ }^{4}$ Even though global infrared intensity is conserved by a sum rule, the apparent depletion of local signal strength can lead to misinterpretation of the results of 1D-IR experiments. The effects of exciton coupling on 1D-IR spectra have been well documented in bulk liquid water, proteins, and DNA; but the influence of the air/water interface on these couplings has yet to be explored..$^{5-11}$

Two-dimensional (2D) vibrational spectroscopic techniques are the only methods immune to this problem because they probe vibrational coupling directly, although the resulting spectra are sometimes too convoluted to analyze. To date, only three studies have investigated vibrational coupling between alkyl chains of a

\footnotetext{
†Contributed equally to this work

*allen@chemistry.ohio-state.edu

*herbert@chemistry.ohio-state.edu
}

surfactant monolayer. ${ }^{12-14}$ Bredenbeck et al. ${ }^{12,13}$ first observed vibrational coupling between the terminal methyl and methylene groups of a dodecanol monolayer at the air/water interface using $2 \mathrm{D}$ vibrational sum frequency generation spectroscopy (VSFG). A wealth of structural information is present within the off-diagonal $\mathrm{C}-\mathrm{H}$ peaks in $2 \mathrm{D}$ vibrational spectra, but accessing many of these details can be unintuitive.

One-dimensional vibrational spectroscopic techniques such as surface-sensitive infrared reflection-absorption spectroscopy (IRRAS) and surface-selective VSFG are simpler to dissect than their 2D analogues and have been much more widely adopted for the analysis of surfactant organization at interfaces. Soluble surfactant adsorption and film surface density are measured through vibrational band peak intensities in which hydrocarbon $\mathrm{C}-\mathrm{H}$ and $\mathrm{C}-\mathrm{D}$ modes are most commonly tracked. ${ }^{15-19}$ The orientation of surfactant terminal methyl groups is frequently assessed via $1 \mathrm{D}$ VSFG and by calculating ratios of $\mathrm{CH}_{3}$ peak intensities obtained by probing the system with different polarizations of light. ${ }^{20,21} \mathrm{An}$ analogous analysis using the $\mathrm{CH}_{2}$ peak intensities from 1D IRRAS spectra is used to assess hydrocarbon chain conformational order at interfaces. ${ }^{22-25} \mathrm{~A}$ method was recently developed to estimate the total intermolecular interactions in a surfactant monolayer at the air/water interface using the intensity ratio amplitude of the second-order Fermi resonance signals generated by VSFG ${ }^{26}$ All of the above analyses rely upon alkyl chain peak intensities, which are susceptible to signal strength reduction via vibrational coupling.

To investigate the influence of vibrational excitons on 1D vibrational spectra, we collected IRRAS spectra and surface tension measurements of perfluorooctanoic acid (PFOA) at the air/water interface and compared the experimental data to theoretical spectra generated from molecular dynamics (MD) simulations combined with an 
$a b$ initio vibrational exciton model (aiVEM). Both theory and experiment reveal signal intensity depletion in the $\mathrm{C}-\mathrm{F}$ vibrational modes at $\mathrm{PFOA}$ concentrations near but below the critical micelle concentration. Signal depletion due to vibrational excitons is also observed in the $\mathrm{C}-\mathrm{H}$ peaks of an insoluble arachidic acid monolayer upon lateral compression, suggesting that the formation of vibrational excitons is a general feature of $1 \mathrm{D}$ vibrational spectra of surfactants at interfaces. This study therefore serves as a cautionary tale against structural analyses using alkyl- and fluoroalkyl-chain vibrational mode peak intensities.

\section{Methods}

A. Chemicals. Pentadecafluorooctanoic acid (PFOA, 96\%, ACROS Organics ${ }^{\mathrm{TM}}$ ) and arachidic acid (AA, $\geq 99 \%$, Sigma-Aldrich) were used as received. All glassware was cleaned in a piranha acid bath. Solutions were prepared in ultrapure water with a resistivity of $18.2 \mathrm{M} \Omega \cdot \mathrm{cm}$ (Milli-Q Advantage A10, EMD Millipore), and $\mathrm{NaCl}$ (Sodium chloride, 99.5\%, for biochemistry, ACROS Organics ${ }^{\mathrm{TM}}$ ) was baked in a furnace at $650^{\circ} \mathrm{C}$ for at least $10 \mathrm{~h}$ to remove residual organic impurities. ${ }^{27}$ PFOA was dissolved in ultrapure water and in an aqueous $0.47 \mathrm{M} \mathrm{NaCl}$ solution, and the $\mathrm{pH}$ of all PFOA solutions was measured at $5.8 \pm 0.1$ due to acidification by atmospheric $\mathrm{CO}_{2}$. AA was dissolved in chloroform (Reagent ACS, 99.8+\%, ACROS Organics ${ }^{\mathrm{TM}}$ ) at $1.0 \mathrm{mM}$. The ultrapure water and $0.47 \mathrm{M}$ $\mathrm{NaCl}$ aqueous solution subphases for AA measurements contained $4 \mu \mathrm{M}$ ethylenediaminetetraacetic acid (EDTA, 99.995\% trace metals basis, Sigma-Aldrich) and were $\mathrm{pH}$ adjusted with sodium hydroxide pellets $(\mathrm{NaOH}, 98 \%$, extra pure, ACROS Organics ${ }^{\mathrm{TM}}$ ) to $\mathrm{pH} 12.5 \pm 0.1$.

B. Surface Tensiometry. PFOA surface tension measurements were collected in borosilicate glass petri dishes using a force tensiometer (Sigma 703D, Biolin Scientific) and the Wilhelmy plate method. A platinum Wilhelmy plate was cleaned with ethanol and ultrapure water and fired until red hot with a Bunsen burner. PFOA solution surfaces were allowed to equilibrate for 3 minutes prior to recording the surface tension, and all measurements were repeated at least five times. Curve fitting of Eq. 2 was performed using the Nonlinear Curve Fit Tool in OriginPro 9.0 (OriginLab 9).

Surface pressure-area isotherms of AA were conducted in triplicate using a Teflon Langmuir trough and Delrin barriers (Biolin Scientific), and surface tension was measured with a platinum Wilhelmy plate. Surface cleanliness, indicated by a surface pressure value $\leq 0.2 \mathrm{mN} / \mathrm{m}$, was assessed by sweeping the barriers across the subphase at the maximum compression speed
(270 mm/min/barrier). AA was spread dropwise onto the aqueous subphase, and the chloroform solvent was allowed to evaporate for 10 minutes. The barriers were symmetrically compressed at a rate of $5 \mathrm{~mm} / \mathrm{min} /$ barrier during the isotherm, and the data were averaged.

\section{Infrared Reflection-Absorption Spectroscopy.} Infrared-reflection absorption spectroscopy (IRRAS) was conducted using a Fourier transform infrared spectrometer (Spectrum 100, PerkinElmer) equipped with a liquid nitrogen-cooled $\mathrm{HgCdTe}(\mathrm{MCT})$ detector. Spectra were collected with unpolarized light in the single-beam mode as an average of 400 scans. The incident beam direction inside the spectrometer was modified by a planar gold mirror at a $48^{\circ}$ angle of incidence relative to surface normal, and the reflected light was redirected toward the detector with a second gold mirror. Energy values were recorded every $0.5 \mathrm{~cm}^{-1}$ between 450 and $4000 \mathrm{~cm}^{-1}$, and the spectral resolution was $4 \mathrm{~cm}^{-1}$. Each experiment was repeated in at least triplicate and analyzed using OriginPro 9.0. For analysis of the PFOA $\mathrm{C}-\mathrm{F}$ vibrational modes, the baseline was subtracted from each spectrum by fitting a line between endpoints 1118 and $1266.5 \mathrm{~cm}^{-1}$, and the peak area of each baseline-subtracted spectrum was integrated between these endpoints. The $\mathrm{C}-\mathrm{H}$ spectral region of AA was baseline-subtracted by fitting a line between endpoints 2800 and $3000 \mathrm{~cm}^{-1}$, and the peak area was integrated between the same endpoints. Figures contain averaged, baseline-subtracted spectra.

D. Molecular Dynamics. AMOEBA parameters for anionic PFOA were obtained using a standard protocol as described in the Supporting Information. Simulations were carried out in the canonical ensemble using slab boundary conditions, with a simulation cell size of $9.5 \times 9.5 \times 29.5 \mathrm{~nm}^{3}$. The temperature was maintained every 0.1 ps at $298 \mathrm{~K}$ using a Bussi thermostat, ${ }^{28}$ with a step size of 2 fs maintained via the RESPA integrator. ${ }^{29}$ All simulations were run using a locally-modified version of Tinker-HP, v. 1.1.30 Our neat water simulations contain a $1: 1$ ratio of $\mathrm{Na}^{+}: \mathrm{PFOA}$ in order to maintain charge neutrality for application of periodic boundary conditions via Ewald summation. $\mathrm{NaCl}$ simulations use a $3: 1$ ratio of $\mathrm{Na}^{+}: \mathrm{PFOA}$ and $2: 1 \mathrm{Cl}^{-}$:PFOA ratio to achieve charge balance, for a net $2: 1$ concentration ratio of $\mathrm{NaCl}: \mathrm{PFOA}$. The net concentration of PFOA was set to 200 molecules in a $9.5 \mathrm{~nm}^{3}$ box, with enough water to provide a density of $\approx 1 \mathrm{~g} / \mathrm{cm}^{3}$.

E. Quantum Chemistry Calculations. Vibrational spectra of PFOA were obtained using a 
vibrational exciton model as described in the Supporting Information. The exciton model requires normal mode frequencies and transition dipole moments for each monomer. These are computed at the EDF2/6-31G(d) level $^{31}$ using the Q-Chem software, v. 5.3, ${ }^{32}$ and scaled by a factor of $0.9805 .{ }^{33}$

\section{Results and Discussion}

A. PFOA Adsorption Measurements. Vibrational exciton formation in soluble surfactant films at the air/aqueous interface of ultrapure $\mathrm{H}_{2} \mathrm{O}$ and $0.47 \mathrm{M}$ $\mathrm{NaCl}$ solution was studied with surface-sensitive IRRAS. The specific concentration of $\mathrm{NaCl}$ was chosen to match the $\mathrm{Na}^{+}$concentration in seawater, and the soluble surfactant PFOA $\left[\mathrm{CF}_{3}\left(\mathrm{CF}_{2}\right)_{6} \mathrm{COOH}\right]$ was selected due to its surface activity and large perfluoroalkyl transition dipole moments. Additionally, PFOA is a strong acid ( $\mathrm{p} K_{\mathrm{a}}<1$ by recent estimates), ${ }^{34,35}$ so the surfactant is fully deprotonated in the bulk and at the surface of ultrapure water $(\mathrm{pH}=5.8)$. IRRAS spectra are analyzed in the $\mathrm{C}-\mathrm{F}$ region $\left(\sim 1100-1300 \mathrm{~cm}^{-1}\right)$ and the spectra are plotted as reflectance-absorbance (RA),

$$
\mathrm{RA}=-\log \left(\frac{R_{\mathrm{m}}}{R_{0}}\right)
$$

where $R_{\mathrm{m}}$ is the reflectivity of the PFOA film and $R_{0}$ is the reflectivity of the aqueous subphase without PFOA.

Three main peaks are present in the $\mathrm{C}-\mathrm{F}$ vibrational mode region of the IRRAS spectra of PFOA in Fig. 1a. (Peak assignments are described in the Supplemental Information.) The peaks red-shift with increasing bulk PFOA concentration, indicative of increasing adsorption and intermolecular interactions between interfacial PFOA molecules. The aqueous subphase composition has a significant impact on the $\mathrm{C}-\mathrm{F}$ peak intensities, lineshapes, and frequencies. For example, $\mathrm{C}-\mathrm{F}$ peaks are not observable for PFOA dissolved in ultrapure water at $2 \mu \mathrm{M}$ or $20 \mu \mathrm{M}$, suggesting that $\mathrm{NaCl}$ enhances PFOA adsorption at low concentrations. Peak integration of the $\mathrm{C}-\mathrm{F}$ vibrational modes (Fig. 1b) further illustrates that $\mathrm{NaCl}$ enhances PFOA adsorption at low concentrations. A PFOA concentration of $200 \mu \mathrm{M}$ brings about an inflection point in this trend wherein the integrated IRRAS peak area of the two aqueous solutions is nearly the same, and at concentrations $>200 \mu \mathrm{M}$ the integrated peak area actually becomes larger in $\mathrm{H}_{2} \mathrm{O}$ than in $0.47 \mathrm{M}$ $\mathrm{NaCl}$.

In response to this apparent deviation from Hofmeister "salting-out" effects, ${ }^{36,37}$ surface tension measurements were collected to further investigate PFOA interfacial adsorption and surface excess for comparison to the IRRAS spectra. Although surface tension does not provide any molecule-specific information, it does yield robust quantitative measurements of surfactant adsorption to the air/water interface. Enhanced surfactant concentration at the surface, relative to the bulk, results in a lower surface tension than that of $\mathrm{H}_{2} \mathrm{O}$ and corresponds to a positive surface excess. The average surface tension of $\mathrm{H}_{2} \mathrm{O}$ was measured at $72.57 \pm 0.22 \mathrm{mN} / \mathrm{m}$ at $21.1 \pm 0.5^{\circ} \mathrm{C}$, consistent with the reference value. ${ }^{38}$

Fig. 1c illustrates a surface tension depression with increasing bulk PFOA concentration. Surface tension measurements for each aqueous subphase solution were fitted to the Szyszkowski equation, ${ }^{39,40}$

$$
\gamma=\gamma_{0}\left[1-a \times \ln \left(\frac{C}{b}+1\right)\right],
$$

where $\gamma$ is the surface tension of the PFOA solution, $\gamma_{0}$ is the surface tension of the aqueous subphase, $C$ is the PFOA concentration in bulk aqueous solution, and $a$ and $b$ are fitted parameters. The results of the fitting (Fig. 1c) show that the magnitude of surface tension depression induced by interfacial PFOA adsorption is highly dependent upon the presence of salt, ${ }^{40-44}$ as the $0.47 \mathrm{M} \mathrm{NaCl}$ solution exhibits decreased surface tension across all bulk PFOA concentrations, relative to $\mathrm{H}_{2} \mathrm{O}$.

To quantify the differences in PFOA surface excess induced by the aqueous subphase, the LangmuirSzyszkowski equation was used to compute the surface excess, $\Gamma:^{40,45}$

$$
\Gamma=\frac{a \gamma_{0}}{R T} \frac{C}{C+b} .
$$

Fitted parameters $a$ and $b$ from Eq. 2 were used in Eq. 3 to obtain the calculated surface excess in Fig. 1d. The aqueous $\mathrm{NaCl}$ solution supports an order of magnitude larger PFOA surface excess than $\mathrm{H}_{2} \mathrm{O}$, with $10-12 \times$ the surface excess of $\mathrm{H}_{2} \mathrm{O}$ for solutions containing $2 \mathrm{mM}$ PFOA and $2 \mu \mathrm{M}$ PFOA, respectively. Second harmonic generation (SHG) spectroscopic measurements of the interfacial electric field (Fig. ??) also confirm enhanced PFOA surface activity in the $\mathrm{NaCl}$ solution, consistent with Hofmeister series "salting-out."

While IRRAS is sensitive to changes in PFOA adsorption due to variable surfactant concentration and aqueous subphase composition, the spectroscopic measurements produce opposite trends relative to the surface tension measurements at concentrations of PFOA exceeding $200 \mu \mathrm{M}$. Surface tensiometry indicates that the $\mathrm{NaCl}$ subphase enhances PFOA surface adsorption relative to $\mathrm{H}_{2} \mathrm{O}$ across all PFOA concentrations, whereas the IRRAS integrated $\mathrm{C}-\mathrm{F}$ peak area implies that $\mathrm{NaCl}$ provides an initial enhancement of PFOA surface adsorption, followed by an anomalous decline in surface adsorption at PFOA concentrations $>200 \mu \mathrm{M}$. The same anomaly was observed for IRRAS and surface tension measurements of PFOA interfacial adsorption to a $10 \mathrm{mM} \mathrm{CaCl}_{2}$ subphase (SI ??), indicating that the discrepancy cannot be ascribed to electrolyte composition nor concentration alone. Aggregation of PFOA could result in $\mathrm{C}-\mathrm{F}$ vibrational coupling, explaining the surfactant concentrationdependent discrepancies between the IRRAS and surface 
a

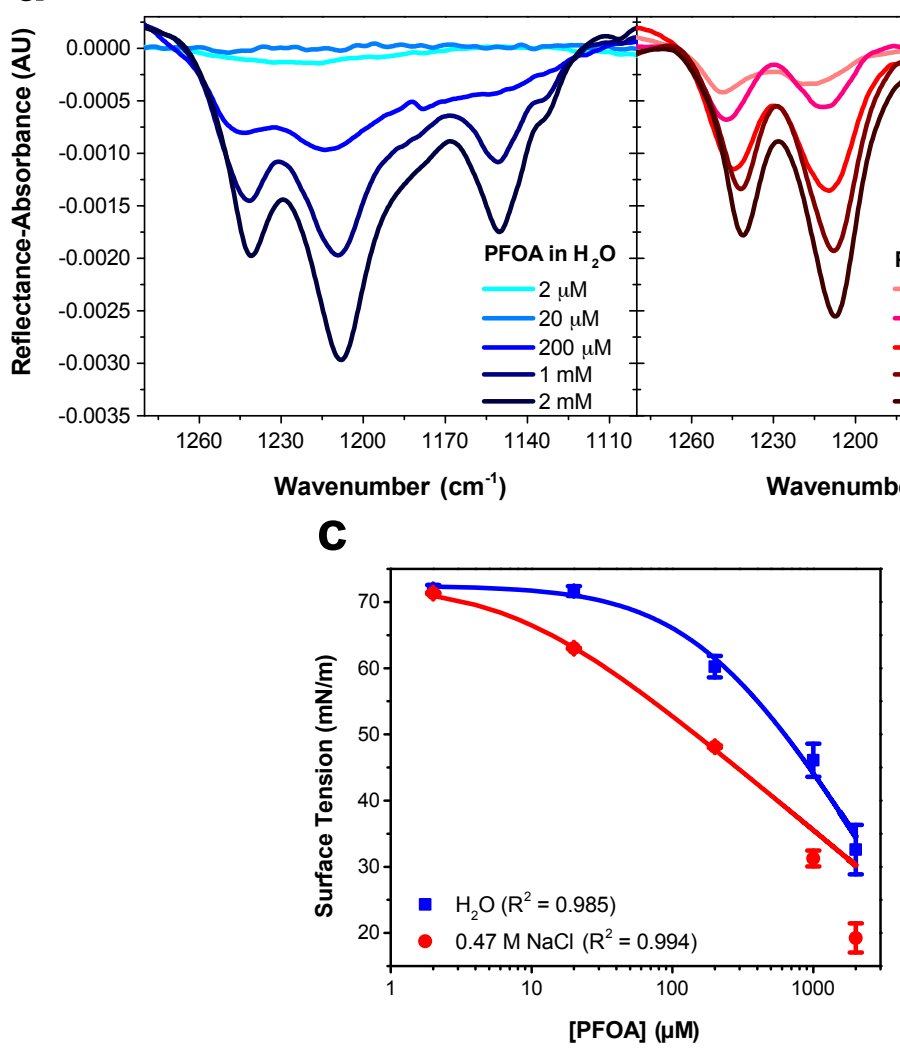

b

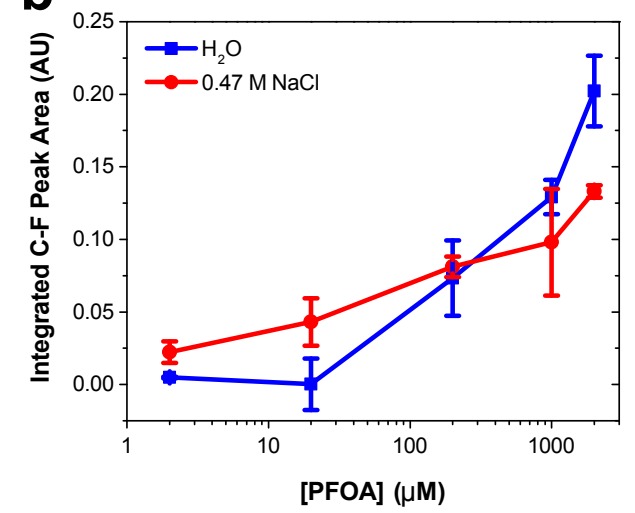

FIG. 1: Perfluorooctanoic acid (PFOA) adsorption to the water and $0.47 \mathrm{M} \mathrm{NaCl(aq)}$ interface. a, Infrared reflectionabsorption spectroscopy (IRRAS) of the C-F vibrational mode region as a function of PFOA bulk concentration. b, Integrated peak area corresponding to the $\mathrm{C}-\mathrm{F}$ vibrational modes as a function of the bulk PFOA concentration. Lines connecting the data points are drawn to guide the eye. c, Surface tension measurements as a function of PFOA bulk concentration. The curved lines connecting the data are fits to the Szyszkowski equation (Eq. 2) and the adjusted $R^{2}$ values are included in the legend. d, The calculated PFOA surface excess from Eq. 3 as a function of bulk concentration. All error bars correspond to one standard deviation from the mean.

tension data. The $0.47 \mathrm{M} \mathrm{NaCl}$ subphase also enhances shoulder features in the IRRAS spectra at $1133 \mathrm{~cm}^{-1}$ and $1105 \mathrm{~cm}^{-1}$, which could be a signature of energy splittings characteristic of vibrational excitons.

In order to prominently exemplify deviations from the Beer-Lambert law, we turn to the effective infrared cross section (Fig. ??). In the absence of vibrational coupling, the integrated peak area would increase linearly with surface excess, allowing for the extraction of an effective infrared cross section from a linear fit of the data. The integrated peak area of PFOA in water is nearly linear with surface excess, suggesting nearly ideal "gas-phase" absorption behavior. However, even in neat water there are deviations from ideal absorption at higher concentrations, suggesting that this phenomenon is concentration dependent. This concentration dependence is amplified in the presence of electrolytes, which induce significant nonlinearity in the $\mathrm{C}-\mathrm{F}$ peak area as a function of PFOA surface excess. Concentration dependence could suggest that the deviations from ideal absorption are caused by PFOA $\cdots$ PFOA interactions. We investigate the origin of these strong deviations from Beer-Lambert absorption in the context of our exciton hypothesis in the next section.

B. Molecular Dynamics Simulations. We begin the theoretical investigation by obtaining reliable structures of PFOA at the air/water interface using classical MD simulations based on the AMOEBA polarizable force field. ${ }^{46}$ The equilibration timescale for aqueous PFOA is on the order of minutes (as implied by the measured surface tension equilibration time), well beyond the timescales accessible to simulation. In view of this, we allowed simulations of the neat water and $\mathrm{NaCl}(\mathrm{aq})$ interfaces to equilibrate for as long as computationally feasible $(2.5 \mathrm{~ns})$ to minimize deviations from equilibrium. (See Sec. 2 for details.)

The radial distribution function ( $\mathrm{RDF}$ ) between the terminal $\left(\mathrm{CF}_{3}\right)$ carbon atoms in PFOA is shown in Fig. 2. The initial peak at $7.5 \AA$ is larger in the $\mathrm{NaCl}$ solution, implying that the PFOA tails are more structured in 


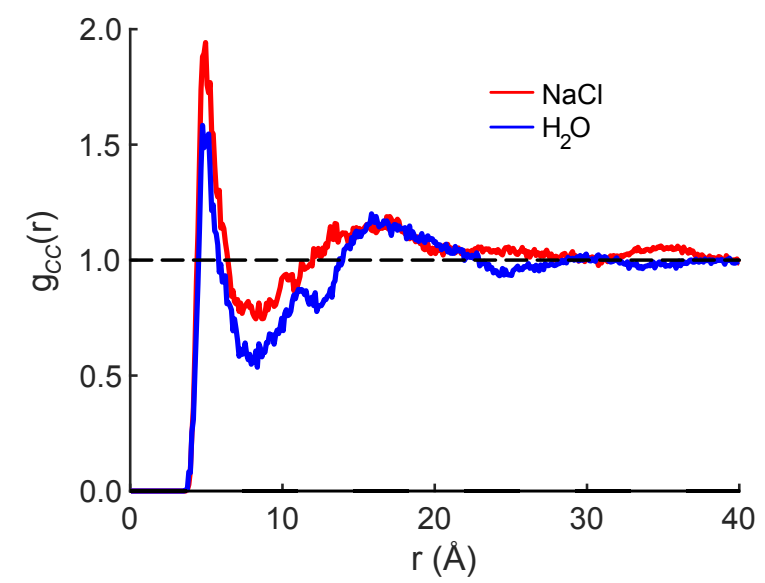

FIG. 2: Radial distribution functions for the terminal $\left(\mathrm{CF}_{3}\right)$ carbon atoms in PFOA.

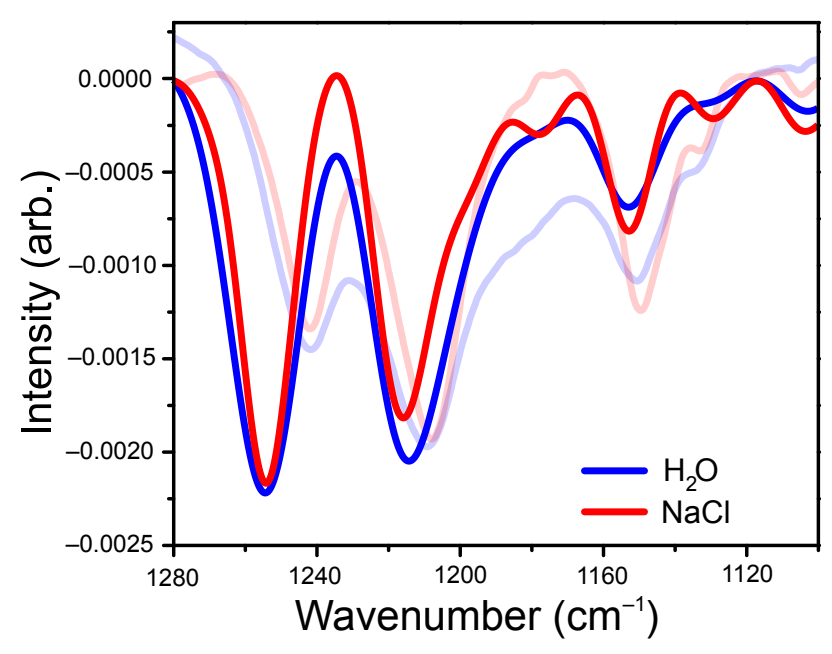

FIG. 3: Exciton model spectra (opaque) overlaid with experimental spectra of $1 \mathrm{mM}$ PFOA (transparent). Each band in the exciton spectra is plotted as a Gaussian lineshape, the width of which is set to the $2 \sigma$ standard deviation in wavenumbers across $25 \mathrm{MD}$ snapshots.

$\mathrm{NaCl}$ solution than in water. Neither our experiments nor simulations show any evidence of micellization, however a higher propensity for soluble surfactants to form micelles in saline solution is well documented, ${ }^{47}$ supporting the notion that PFOA molecules aggregate more closely in the presence of $\mathrm{NaCl}$. While we are not expressly concerned with micellization, the propensity for molecules to self-aggregate has important consequences for delocalization of the vibrational wavefunctions, as the vibrational couplings decay rapidly $\left(\sim R^{-3}\right)$ with distance.

C. Vibrational Exciton Model Spectrum. We modeled vibrational excitons following theoretical groundwork laid by others, ${ }^{4,48,49}$ parameterizing our model with $a b$ initio harmonic vibrational frequencies for PFOA, taken from 25 snapshots spanning 250 ps of MD simulation. We couple every vibrational mode of all 200 PFOA molecules at each snapshot via the $a b$ initio-derived transition dipole moments; see Supplementary Information for details. Non-interacting vibrational modes of each PFOA molecule are computed in vacuum and the couplings are computed without dielectric screening, yielding a final spectrum that reflects the intermolecular coupling of gas-phase PFOA monomers within a solution-phase distribution of positions. Solvent effects are omitted for simplicity, computational efficiency, and to gauge whether they are needed to reproduce the experimental spectrum. Vibrational coupling to the solvent is frequently invoked in cases of odd or difficult-to-explain spectral features, and use of a minimalist model allows us to probe whether coupling to the solvent is actually important.

The $a i$ VEM spectra in Fig. 3 show exceptionally good agreement with experiment. All center wavelengths are in quantitative agreement (Table I). Aside from the overestimate of the intensity of the high-energy band, the exciton model spectrum does a remarkable job of reproducing the fine details of the experimental spectrum, including asymmetry of the central band and the appearance of shoulders at 1180,1130 , and $1105 \mathrm{~cm}^{-1}$ that are all more pronounced in $\mathrm{NaCl}$ than in water. Importantly, the loss of intensity upon introduction of $\mathrm{NaCl}$ that is observed in the integrated peak areas is reproduced in the exciton model spectrum.

Omission of solvent effects in the $a i \mathrm{VEM}$ allows us to isolate the origins of several important factors that influence the IRRAS spectra of PFOA. First, the appearance of shoulders in the $\mathrm{NaCl}$ spectrum is characteristic of exciton splitting and emerges due to vibrational coupling between $\mathrm{C}-\mathrm{F}$ modes. These shoulders reflect a smearing out of the vibrational density of states by virtue of delocalization of the vibrational wavefunction. This means that vibrational bands with nearby shoulders (e.g., the center band at $\sim 1210 \mathrm{~cm}^{-1}$ and the low-energy band at $\sim 1150 \mathrm{~cm}^{-1}$ ) couple most strongly, as indicated also by their more dramatic changes in intensity between subphases. The intensity of the high-energy band changes the least between subphases, and does not appear to split or give rise to shoulders. This implies that the highenergy modes are spectators to the exciton delocalization. Emergence of shoulders and signal depletion in the experimental $\mathrm{NaCl}$ spectrum can both be attributed directly to vibrational excitons.

Combining the structural insights from MD simulations, experimental surface tension measurements, and the agreement of the $a i \mathrm{VEM}$ with experiment, the vibrational exciton hypothesis appears to be more likely than desorption of PFOA back into the aqueous subphase. This hypothesis explains the anomalous phenomena that are observed in IRRAS measurements of other soluble surfactants such as sodium dodecyl sulfate, where similar intensity decreases have been noted in the spectra of the $\mathrm{C}-\mathrm{H}$ tails. ${ }^{15} \mathrm{An}$ important question remains as to 
TABLE I: Center Wavenumbers (in $\mathrm{cm}^{-1}$ ) of Major Features in IRRAS Spectra of PFOA.

\begin{tabular}{|c|c|c|c|c|c|c|c|c|c|}
\hline \multirow{2}{*}{ Subphase } & \multicolumn{3}{|c|}{$\omega_{1}$} & \multicolumn{3}{|c|}{$\omega_{2}$} & \multicolumn{3}{|c|}{$\omega_{3}$} \\
\hline & aiVEM & Expt. & $\overline{\Delta^{a}}$ & aiVEM & Expt. & $\overline{\Delta^{a}}$ & aiVEM & Expt. & $\Delta^{a}$ \\
\hline $\mathrm{H}_{2} \mathrm{O}$ & 1254.0 & 1241.7 & 12.3 & 1214.0 & 1209.5 & 4.5 & 1153.0 & 1150.5 & 2.5 \\
\hline $\mathrm{NaCl}$ & 1254.0 & 1242.0 & 12.0 & 1216.0 & 1208.0 & 8.0 & 1153.0 & 1149.7 & 3.3 \\
\hline
\end{tabular}

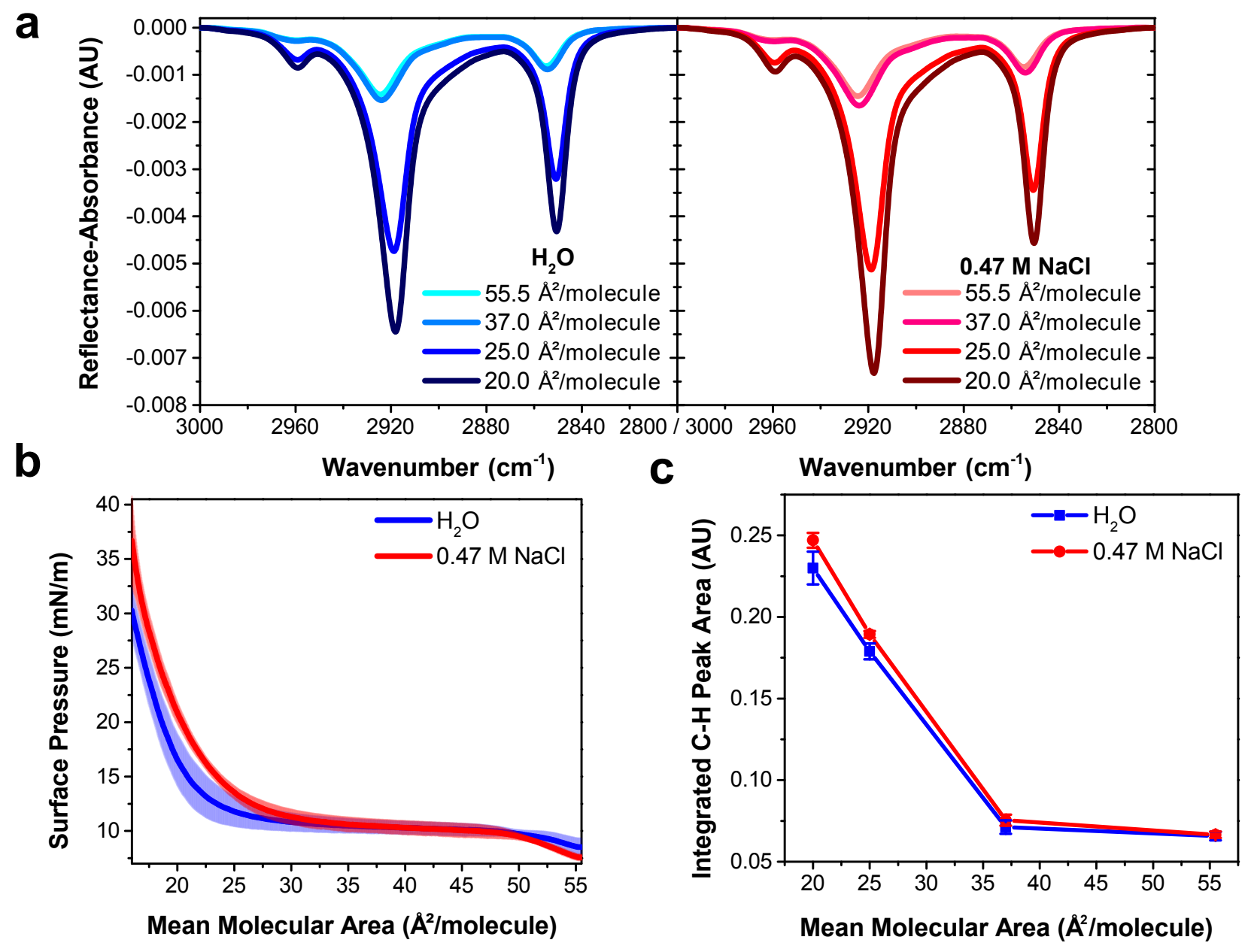

FIG. 4: Deprotonated arachidic acid (AA) monolayers on water and $0.47 \mathrm{M} \mathrm{NaCl}$ at pH 12.5 . Both aqueous subphase solutions contain $4 \mu \mathrm{M}$ ethylenediaminetetraacetic acid. a, Infrared reflection-absorption spectroscopy (IRRAS) of the AA monolayer C-H stretching region as a function of mean molecular area, expressed in units of $\AA^{2} /$ molecule. b, Surface pressure-area isotherms of AA monolayers. Shading represents one standard deviation from the mean. c, Integrated peak area corresponding to $\mathrm{C}-\mathrm{H}$ vibrational modes as a function of AA monolayer mean molecular area. Lines connecting the data points are drawn to guide the eye, and error bars represent one standard deviation from the mean.

whether vibrational excitons are unique to soluble surfactants, or if this phenomenon is generalizable to insoluble monolayers. This is addressed below.

The impressive agreement of the $a i \mathrm{VEM}$ with the PFOA experiments suggests that it is a useful tool with predictive capacity, but the $\mathrm{C}-\mathrm{F}$ bonds in PFOA are rather exotic, making them poor surrogates for general trends in self-aggregating lipids at interfaces. As a model of the latter, we applied the aiVEM to a $2 \mathrm{D}$ sheet of 24 uniformly spaced octanoic acid $\left(\mathrm{C}_{8} \mathrm{H}_{16} \mathrm{O}_{2}\right)$ molecules, in an effort to hypothesize about the role of vibrational coupling in the $1 \mathrm{D}$ spectroscopy of $\mathrm{C}-\mathrm{H}$ modes in an ordered monolayer at the air/water interface. The results (Fig. ??) feature a lowering in the local intensity of the $\mathrm{C}-\mathrm{H}$ vibrational modes upon compression from a diffuse monolayer to one corresponding to $5 \AA$ mean molecular area. The prediction that vibrational coupling can induce distance-dependent signal reduction for an insoluble monolayer is next examined experimentally. 
D. Arachidic Acid Monolayer Measurements. To determine if vibrational excitons can be observed in $1 \mathrm{D}$ vibrational spectroscopy of surfactants more generally, an insoluble arachidic acid $\left(\mathrm{AA}, \mathrm{C}_{20} \mathrm{H}_{40} \mathrm{O}_{2}\right)$ monolayer was probed with IRRAS and surface tensiometry. AA forms a stable monolayer at the air/water interface (Fig. ??), even when over $95 \%$ of the carboxylic acid headgroups are deprotonated at $\mathrm{pH} \sim 12,{ }^{50}$ making it an excellent insoluble surfactant to contrast with PFOA. IRRAS spectra of an AA monolayer on water and on $0.47 \mathrm{M} \mathrm{NaCl}$ at $\mathrm{pH} 12.5$ were collected at constant mean molecular area, and a plot of the $\mathrm{C}-\mathrm{H}$ vibrational modes is shown in Fig. 4a. To prevent trace metal binding to the AA monolayer, $4 \mu \mathrm{M}$ ethylenediaminetetraacetic acid was added to both subphase solutions. ${ }^{51}$

Mean molecular area values were chosen based on the surface pressure-area isotherms in Fig. 4b. At $55.5 \AA^{2} /$ molecule, the AA monolayer is in a liquid expanded (LE) phase in which the surfactants are fluid and not yet aggregated. Monolayer compression yields a plateau region $\left(37 \AA^{2} /\right.$ molecule) corresponding to a first-order phase transition between the LE and tilted condensed (TC) phases, known as the LE-TC coexistence region. ${ }^{51,52} \mathrm{AA}$ surfactants aggregate into $2 \mathrm{D}$ domains at the air/water interface and increase in size with compression. ${ }^{53}$ (Brewster angle microscopy images of the AA domain morphologies are shown in Fig. ??.) The monolayer begins to transition to the TC phase starting at $25 \AA^{2} /$ molecule and the transition is complete by $20 \AA^{2} /$ molecule, characterized by a condensed sheet of surfactant. The AA monolayer has a higher surface pressure at mean molecular areas $\lesssim 35 \AA^{2} /$ molecule on the $\mathrm{NaCl}$ solution subphase, indicating that $\mathrm{NaCl}$ expands the monolayer. The chloride counterion is likely to blame for this monolayer expansion, as it has a greater propensity to partition to the air/water interface than sodium, whereupon it intercalates between the carboxylic acid head groups of AA, amplifying electrostatic repulsion. ${ }^{54,55}$

The three peaks in the IRRAS spectra of AA (Fig. 4a) correspond to the $v_{\mathrm{AS}}-\mathrm{CH}_{3}$ stretch at $2959 \mathrm{~cm}^{-1}$, the $v_{\mathrm{AS}}-\mathrm{CH}_{2}$ stretch at $2918 \mathrm{~cm}^{-1}$, and the $v_{\mathrm{S}^{-}} \mathrm{CH}_{2}$ stretch at $2850.5 \mathrm{~cm}^{-1} .{ }^{56}$ A mutual feature of these spectra (regardless of subphase identity) is that the region between the two high-energy bands becomes more intense as a function of monolayer compression. This feature is indicative of delocalization of the vibrational wavefunction across a new region in the vibrational manifold, hinting at exciton splitting. At smaller mean molecular area (25.0 and $20.0 \AA^{2} /$ molecule), the $\mathrm{C}-\mathrm{H}$ peak intensity is greater on $0.47 \mathrm{M} \mathrm{NaCl}$ than on $\mathrm{H}_{2} \mathrm{O}$, as shown in the integrated $\mathrm{C}-\mathrm{H}$ peak areas in Fig. 4d. As the AA monolayer is compressed to smaller mean molecular area, the $\mathrm{C}-\mathrm{H}$ peak area of $\mathrm{AA}$ on the $\mathrm{NaCl}$ solution subphase becomes larger relative to the water subphase. AA surfactants are closer together on the water subphase and therefore subject to stronger vibrational couplings, which decay with distance as $R^{-3}$. As a result, excitonic behavior is observable in insoluble surfactant monolayers via IRRAS signal intensity depletion in the $\mathrm{C}-\mathrm{H}$ stretching region.

The observable impact of vibrational excitons on the IRRAS spectra of AA monolayers is smaller in magnitude than that of PFOA. Our calculations imply that, on average, transition dipole moments of $\mathrm{C}-\mathrm{F}$ moieties are $3 \times$ larger than those of $\mathrm{C}-\mathrm{H}$ moieties in fatty acids of commensurate chain length, contributing to the observed differences in vibrational coupling. Soluble surfactants are able to access an extra degree of freedom (in comparison to insoluble surfactants that are restricted to the interfacial plane), which could also influence the vibrational mode coupling by allowing for three-dimensional aggregation.

E. Observable Delocalization Length. In order to develop a notion of how delocalized the vibrational wavefunction becomes at the air/water interface, we turn to a $2 \mathrm{D}$ sheet model of AA. While we report data for a $2 \mathrm{D}$ sheet and 3D unit cell models of PFOA, there are several factors that make AA a cleaner model to study. First, the surface presence of AA is well quantified experimentally, and the monolayer shows no evidence of desorption into the aqueous subphase. Second, the effects of dielectric screening between the surfactants can be neglected, as the intermolecular coupling between AA tails is easily assumed to be far removed from the large dielectric of the aqueous medium below. Lastly, the mean molecular area is well known experimentally, and thus the intermolecular separation can be readily transferred to a theoretical model.

In order to quantify the extent of vibrational wavefunction delocalization, Fig. 5 plots the exciton splitting $\left(\omega_{+}-\omega_{-}\right)$for the vibrational mode at $2918 \mathrm{~cm}^{-1}$ as a function of intermolecular separation along a uniform expansion coordinate of a $2 \mathrm{D}$ sheet model of AA. Fundamentally, the splittings in the exciton model are the quantum mechanical tunneling splittings in multiplefinite-well potentials, which fall off exponentially with distance. Therefore, we fit a biexponential function $A \exp (-\alpha r)+B \exp (-\beta r)$ where $A$ and $B$ are normalization constants and $\alpha$ and $\beta$ are related to the tunneling length scales at short range and long range, respectively. In the short range, the Coulomb potential between classical dipoles diverges, hence the long-range portion of the fit represents classical dipole physics.

The result of the biexponential fit $(A=536.7, B=$ 8.739, $\alpha=1.326 \AA^{-1}$, and $\left.\beta=0.052 \AA^{-1}\right)$ is shown in Fig. 5a. The long-range exponential function determines the delocalization length, and integration suggests that $90 \%$ of the wavefunction is contained in the relatively large delocalization length of $23 \AA$ (which is still an order of magnitude smaller than delocalization length scales reported in solids). ${ }^{3}$ Despite the sizable delocalization length, the differences in the splittings as a function of mean molecular area do not become particularly pronounced until after the monolayer is compressed into the 

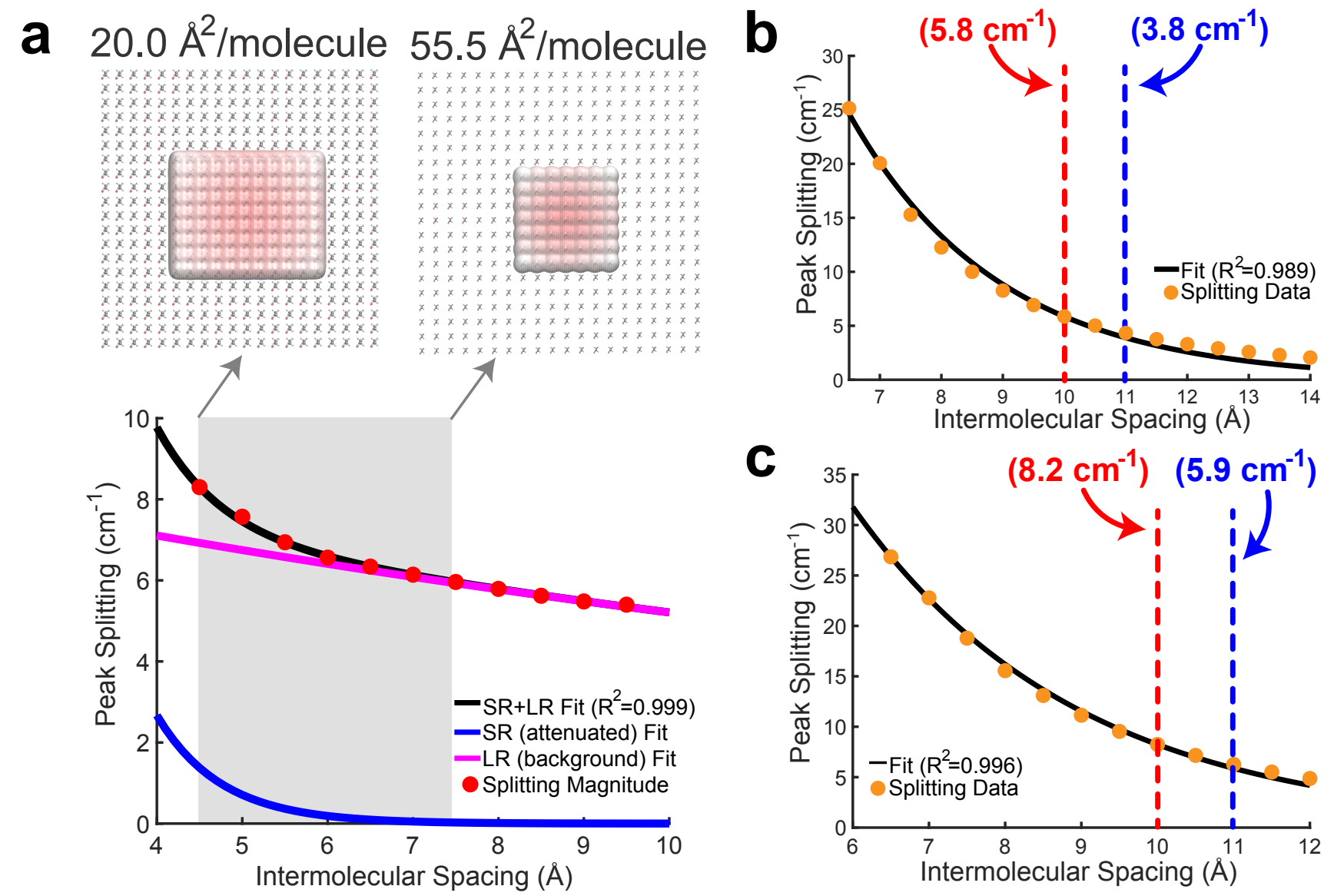

FIG. 5: Delocalization length models: (a) A biexponential fit of AA exciton (tunneling) splittings for the $2918 \mathrm{~cm}^{-1}$ mode as a function of intermolecular spacing in a model system of 100 AA molecules. The shaded region corresponds to the experimentally probed mean molecular area values. The extent of wavefunction delocalization is visualized at the experimental minimum and maximum mean molecular area values. (b) An exponential fit of exciton splittings of the $1213 \mathrm{~cm}^{-1}$ mode for a $(10 \times 10) 2 \mathrm{D}$ PFOA sheet model along a uniform compression coordinate. (c) An exponential fit of exciton splittings of the $1213 \mathrm{~cm}^{-1}$ mode $^{-1}$ for a $(5 \times 5 \times 5) 3 \mathrm{D}$ PFOA unit cell with randomly generated PFOA orientations along a uniform compression coordinate. In (b) and (c), the red and blue dashed lines correspond to the nearest neighbor distances from the integrated $\mathrm{g}(\mathbf{r})$ for $\mathrm{NaCl}$ and $\mathrm{H}_{2} \mathrm{O}$, respectively, and each is annotated with the tunneling splitting at that intermolecular spacing.

region governed by the short-range exponential, $\sim 6 \AA$ (36 $\AA^{2}$ /molecule). Interestingly, this is precisely consistent with experiment, where the differences in integrated $\mathrm{C}-\mathrm{H}$ peak intensities are not pronounced until a compression of $25 \AA^{2} /$ molecule. Experimentally, we have shown that the intermolecular spacing on the $0.47 \mathrm{M}$ $\mathrm{NaCl}$ subphase is more diffuse than on neat water, and the influence of vibrational wavefunction delocalization is exponentially more pronounced as a function of the intermolecular spacing in the short range. Our model shows that this exponential is governed by a rather large coefficient, suggesting that these subtle changes in the intermolecular packing between the two subphases will cause large enough differences in the measured intensities to be noticeable in 1D IRRAS spectra.

The PFOA models were studied at larger intermolecular spacings, and therefore required only a simple exponential fit $(A \exp [-\alpha r])$, as the dipole-dipole potential is not divergent at long range. The results of these fits for a 2D sheet model (Fig. 5b) and a 3D unit cell in which the orientations of the PFOA molecules were generated via the application of random rotations (Fig. 5c) are consistent with one another despite differences in orientation. The normalization parameters are $A=354.3$ for the $2 \mathrm{D}$ sheet and $A=240.8$ for the $3 \mathrm{D}$ cell. The differences in normalization are expected, as the systems have different dimensionality and contain different numbers of molecules. The interesting number is the tunneling coefficient, where $\alpha=0.41 \AA^{-1}$ for the $2 \mathrm{D}$ sheet and $\alpha=0.34 \AA^{-1}$ for the 3D unit cell. The tunneling coefficients (and the splitting magnitudes themselves) are very similar between models, suggesting that the major difference between the two is simply a shift to longer delocalization lengths due to the stronger interactions in the 3D unit cell. As expected, the additional degree of freedom of translational motion amplifies interactions between 
transition dipole moments simply by nature of there being more nearby interactions per PFOA monomer. In comparison with the AA data, the exciton splittings in PFOA are larger due to the significantly larger transition dipole moment of the PFOA transition $(0.5 \mathrm{D})$ relative to the AA transition (0.25 D). However, due to transition dipole moment alignment in the more ordered $2 \mathrm{D}$ sheet motif, along with less intermodal transition dipole cancellation due to the more ordered molecular geometry of AA (staggered rather than helical), the AA delocalization length is much larger, as $\beta=0.05 \AA^{-1} \ll \alpha=0.4 \AA^{-1}$. Therefore the exciton splitting in PFOA is larger within the space where PFOA molecules interact, and the interactions are supplemented by the partial solubility of PFOA, but they are more short-ranged than in their hydrocarbon counterparts.

In terms of the measurable impact on the 1D IRRAS spectra, our models corroborate the experimental data quite nicely. Although the delocalization is far more significant in AA, the subtle differences in packing structure at the interface due to the composition of the subphase will only alter the vibrational exciton splittings by fractions of a wavenumber per vibrational mode. The result is an effect that is noticeable, but subtle. As for the PFOA spectra, the tunneling splittings that result from differences in subphase composition are augmented by the 3D nature of the PFOA interactions and yield far more significant multiple wavenumber splittings, leading to a more stark decline in the local intensity. The combination of theory and experiment presented here strongly suggests that vibrational wavefunction delocalization is to blame for the perceived signal losses in 1D IRRAS of surfactants at the air/water interface.

\section{Conclusions}

We observed the signature of vibrational excitons in surfactants at the air/water interface via alkyl and fluoroalkyl vibrational mode signal reduction in 1D surface-sensitive infrared reflection-absorption spectroscopy (IRRAS). Surface tensiometry, molecular dynamics simulations, and ab initio calculations confirm the existence of significant vibrational coupling in both soluble fluoroalkyl and insoluble alkyl surfactants. Intentionally excluding solvent effects from the theoretical model reveals that coupling with the solvent is unimportant in obtaining the most pertinent features of the IRRAS spectrum. Instead, model IRRAS spectra of perfluorooctanoic acid obtained from molecular dynamics simulations and vibrational exciton calculations agree nearly quantitatively with experiment, despite exclusion of solvent effects, indicating that surfactant intermolecular coupling dominates the observed spectral features. While solvent effects are minimal, the ionic composition of the aqueous phase plays a significant role in modulating vibrational coupling via the strong $R^{-3}$ distance dependence of transition-dipole interactions. Salts mod- ulate intermolecular distances (and therefore vibrational couplings) between surfactants, depleting infrared intensity of 1D surface-sensitive spectra. In light of this new interpretation, 1D vibrational spectroscopic studies of surfactants at surfaces of varying aqueous phase compositions should be carefully scrutinized.

The results described above present both challenges and opportunities for the surface science community. Quantitative analyses involving vibrational mode peak intensities must be approached with caution to avoid misinterpretation of vibrational spectra due to vibrational coupling. The molecular environment has a profound impact on the organization of self-aggregating molecules, making fluid interfaces particularly susceptible to the influence of vibrational excitons. The emergence of vibrational excitons in 1D spectra offers a new, highly-sensitive spectroscopic handle for probing molecular organization at interfaces. Further study of the spectral perturbations induced by vibrational excitons is needed for the development of improved 1D surface-sensitive vibrational spectroscopic analyses. More broadly, the influence of vibrational excitons should be investigated in systems beyond the air/water interface, particularly in confined environments like aerosols.

\section{Supporting Information}

Additional supporting data from the experiments and details of the theoretical model.

\section{Acknowledgements}

K.A.C.-F., M.E.F., and H.C.A. acknowledge funding from the National Science Foundation Center for Aerosol Impacts on Chemistry of the Environment (NSF-CAICE) under Award No. CHE-1801971. K.C.-F. and J.M.H. acknowledge funding from the National Science Foundation under Award No. CHE-1955282. Calculations were performed at the Ohio Supercomputer Center under project no. PAA-0003. K.C.-F. acknowledges a Presidential Fellowship from The Ohio State University. The authors thank Ka Chon Ng for assistance with the SHG measurements.

\section{References}

1 Baumler, S. M.; Allen, H. C. Vibrational spectroscopy of gas-liquid interfaces. In Physical Chemistry of Gas-Liquid Interfaces, Vol. 1; Faust, J. A.; House, J. E., Eds.; Elsevier: Amsterdam, 2018; Chapter 5, pages 105-133.

2 Israelachvili, J. N. Intermolecular and Surface Forces; Academic Press: Amsterdam, 3rd ed.; 2011.

3 Muller, E. A.; Gray, T. P.; Zhou, Z.; Cheng, X.; Khatib, O.; Bechtel, H. A.; Raschke, M. B. Vibrational 
exciton nanoimaging of phases and domains in porphyrin nanocrystals. Proc. Natl. Acad. Sci. USA 2020, 117, 70307037.

4 Signorell, R. Verification of the vibrational exciton approach for $\mathrm{CO}_{2}$ and $\mathrm{N}_{2} \mathrm{O}$ nanoparticles. J. Chem. Phys. 2003, 118, 2707-2715.

5 Torii, H. Extent of delocalization of vibrational modes in liquids as a result of competition between diagonal disorder and off-diagonal coupling. J. Phys. Chem. A 2004, 108, 2103-2107.

6 Torii, H. Intra- and intermolecular charge fluxes induced by the $\mathrm{OH}$ stretching mode of water and their effects on the infrared intensities and intermolecular vibrational coupling. J. Phys. Chem. B 2010, 114, 13403-13409.

7 Auer, B. M.; Skinner, J. L. Dynamical effects in line shapes for coupled chromophores: Time-averaging approximation. J. Chem. Phys. 2007, 127, 104105.

8 Auer, B. M.; Skinner, J. L. IR and Raman spectra of liquid water: Theory and interpretation. J. Chem. Phys. 2008, 128, 224511.

9 Yang, M.; Skinner, J. L. Signatures of coherent vibrational energy transfer in IR and Raman line shapes for liquid water. Phys. Chem. Chem. Phys. 2010, 12, 982-991.

10 Krummel, A. T.; Zanni, M. T. DNA vibrational coupling revealed with two-dimensional infrared spectroscopy: Insight into why vibrational spectroscopy is sensitive to DNA structure. J. Phys. Chem. B 2006, 110, 13991-14000.

11 Krummel, A. T.; Zanni, M. T. Evidence for coupling between nitrile groups using DNA templates: A promising ew method for monitoring structures with infrared spectroscopy. J. Phys. Chem. B 2008, 112, 1136-1338.

12 Bredenbeck, J.; Ghosh, A.; Smits, M.; Bonn, M. Ultrafast two dimensional-infrared spectroscopy of a molecular monolayer. J. Am. Chem. Soc. 2008, 130, 2152-2153.

13 Bredenbeck, J.; Ghosh, A.; Nienhuys, H.-K.; Bonn, M. Interface-specific ultrafast two-dimensional vibrational spectroscopy. Acc. Chem. Res. 2009, 42, 1332-1342.

14 Lackner, M.; Hille, M.; Hasselbrink, E. Vibrational energy redistribution between $\mathrm{CH}$ stretching modes in alkyl chain monolayers revealed by time-resolved two-color pumpprobe sum frequency spectroscopy. J. Phys. Chem. Lett. 2020, 11, 108-112.

15 Prosser, A. J.; Franses, E. I. Infrared reflection absorption spectroscopy (IRRAS) of aqueous nonsurfactant salts, ionic surfactants, and mixed ionic surfactants. Langmuir 2002, 18, 9234-9242.

16 Kawai, T.; Kamio, H.; Kondo, T.; Kon-No, K. Effects of concentration and temperature on SDS monolayers at the air-solution interface studied by infrared external reflection spectroscopy. J. Phys. Chem. B 2005, 109, 44974500 .

17 Rao, Y.; Li, X.; Lei, X.; Jockusch, S.; George, M. W.; Turro, N. J.; Eisenthal, K. B. Observations of interfacial population and organization of surfactants with sum frequency generation and surface tension. J. Phys. Chem. C 2011, 115, 12064-12067.

18 Asadzadeh Shahir, A.; Nguyen, K. T.; Nguyen, A. V. A sum-frequency generation spectroscopic study of the Gibbs analysis paradox: Monolayer or sub-monolayer adsorption? Phys. Chem. Chem. Phys. 2016, 18, 8794-8805.

19 Luo, M.; Wauer, N. A.; Angle, K. J.; Dommer, A. C.; Song, M.; Nowak, C. M.; Amaro, R. E.; Grassian, V. H. Insights into the behavior of nonanoic acid and its conjugate base at the air/water interface through a combined experimental and theoretical approach. Chem. Sci. 2020, 11, 10647-10656.

20 Wang, H.-F.; Gan, W.; Lu, R.; Rao, Y.; Wu, B.-H. Quantitative spectral and orientational analysis in surface sum frequency generation vibrational spectroscopy (SFGVS). Int. Rev. Phys. Chem. 2005, 24, 191-256.

21 Rudd, B. A. W.; Vidalis, A. S.; Allen, H. C. Thermodynamic versus non-equilibrium stability of palmitic acid monolayers in calcium-enriched sea spray aerosol proxy systems. Phys. Chem. Chem. Phys. 2018, 20, 1632016332 .

22 Gericke, A.; Hühnerfuss, H. In situ investigation of saturated long-chain fatty acids at the air/water interface by external infrared reflection-absorption spectrometry. J. Phys. Chem. 1993, 97, 12899-12908.

23 ad J. W. Brauner, R. M.; Gericke, A. External infrared reflection absorptiion spectrometry of monolayer films at the air-water interface. Annu. Rev. Phys. Chem. 1995, 46, 305-334.

24 Buontempo, J. T.; Rice, S. A. Infrared external reflection spectroscopic studies of phase transitions in Langmuir monolayers of heneicosanol. J. Chem. Phys. 1993, 98, 5835-5846.

25 Cheng, S.; Du, L.; George, C. Understanding the interfacial behavior of typical perfluorocarboxylic acids at surfactant-coated aqueous interfaces. J. Geophys. Res.Atmos. 2020, 125, e2019JD032182.

26 Tian, K.; Zhang, B.; Ye, S.; Luo, Y. Intermolecular interactions at the interface quantified by surface-sensitive second-order Fermi resonant signals. J. Phys. Chem. C 2015, 119, 16587-16595.

27 Hua, W.; Verreault, D.; Adams, E. M.; Huang, Z.; Allen, H. C. Impact of salt purity on interfacial water organization revealed by conventional and heterodynedetected vibrational sum frequency generation spectroscopy. J. Phys. Chem. C 2013, 117, 19577-19585.

28 Bussi, G.; Donadio, D.; Parrinello, M. Canonical sampling through velocity rescaling. J. Chem. Phys. 2007, 126, 014101.

29 Tuckerman, M.; Berne, B. J. Reversible multiple time scale molecular dynamics. J. Chem. Phys. 1992, 97, 1990-2001.

30 Lagardère, L.; Jolly, L.-H.; Lipparini, F.; Aviat, F.; Stamm, B.; Jing, Z. F.; Harger, M.; Torabifard, H.; Cisneros, G. A.; Schneiders, M. J.; Gresh, N.; Maday, Y.; Ren, P. Y.; Ponder, J. W.; Piquemal, J.-P. Tinker-HP: A massively parallel molecular dynamics package for multiscale simulations of large complex systems with advanced point dipole polarizable force fields. Chem. Sci. 2018, 9, 956-972.

31 Lin, C. Y.; George, M. W.; Gill, P. M. W. EDF2: A density functional for predicting molecular vibrational frequencies. Aust. J. Chem. 2004, 57, 365-370.

32 Shao, Y. et al. Advances in molecular quantum chemistry contained in the Q-Chem 4 program package. Mol. Phys. 2015, 113, 184-215.

33 Merrick, J. P.; Moran, D.; Radom, L. An evaluation of harmonic vibrational frequency scaling factors. J. Phys. Chem. A 2007, 111, 11683-11700.

34 Vierke, L.; Berger, U.; Cousins, I. T. Estimation of the acid dissociation constant of perflouroalkyl carboxylic acids through an experimental investigation of their waterto-air transport. Environ. Sci. Technol. 2013, 47, 1103211039 .

35 Johansson, J. H.; Yan, H.; Berger, U.; Cousins, I. T. 
Water-to-air transfer of branched and linear PFOA: Influence of pH, concentration and water type. Emerging Contaminants 2017, 3, 46-53.

${ }^{36}$ Kalra, A.; aand S. M. Cramer, N. T.; Garde, S. Saltingin and salting-out of hydrophobic solutes in aqueous salt solutions. J. Phys. Chem. B 2001, 105, 6380-6386.

37 Zhao, X.; Nathanson, G. M.; Andersson, G. G. Experimental depth profiles of surfactants, ions, and solvent at the Angstrom scale: Studies of cationic and anionic surfactants and their salting out. J. Phys. Chem. B 2020, 124, 2218-2229.

38 Vargaftik, N. B.; Volkov, B. N.; Voljak, L. D. International tables of the surface tension of water. J. Phys. Chem. Ref. Data 1983, 12, 817-820.

39 von Szyszkowski, B. Experimentelle Studien über kapillare Eigenschaften der wässerigen Lösungen von Fettsäuren. Z. Phys. 1908, 64, 385-414.

40 Costanza, J.; Arshadi, M.; Abriola, L. M.; Pennell, K. D. Accumulation of PFOA and PFOS at the air-water interface. Environ. Sci. Technol. Lett. 2019, 6, 487-491 Erratum: Environ. Sci. Technol. Lett. 2020, 7, 446.

41 Shinoda, K.; Nakayama, H. Separate determinations of the surfaces excesses of surface-active ions and of gegenions at the air-solution interface. J. Colloid Sci. 1963, 18, 705712 .

42 Shinoda, K.; Hato, M.; Hayashi, T. Physicochemical properties of aqueous solutions of fluorinated surfactants. J. Phys. Chem. 1972, 76, 909-914.

43 Downes, N.; Ottewill, G. A.; Ottewill, R. H. An investigation of the behaviour of ammonium perfluoro-octanoate at the air/water interface in the absence and presence of salts. Colloids Surf. A 1995, 102, 203-211.

44 Brusseau, M. L.; Van Glubt, S. The influence of surfactant and solution composition on PFAS adsorption at fluid-fluid interfaces. Water Res. 2019, 161, 17-26.

45 Langmuir, I. The constitution and fundamental properties of solids and liquids. II. Liquids. J. Am. Chem. Soc. 1917, 39, 1848-1906.

46 Ponder, J. W.; Wu, C.; Ren, P.; Pande, V. S.; Chodera, J. D.; Schnieders, M. J.; Haque, I.; Mobley, D. L.; Lambrecht, D. S.; R. A. DiStasio, J.; HeadGordon, M.; Clark, G. N. I.; Johnson, M. E.; HeadGordon, T. Current status of the AMOEBA polarizable force field. J. Phys. Chem. B 2010, 114, 2549-2564.

47 Butt, H.-J.; Graf, K.; Kappl, M. Physics and Chemistry of Interfaces; Wiley-VCH: Weinheim, 3rd ed.; 2003.

${ }^{48}$ Hexter, R. M. Intermolecular coupling of vibrations in molecular crystals: A vibrational exciton approach. J. Chem. Phys. 1960, 33, 1833-1841.

49 Persson, B. N. J.; Ryberg, R. Vibrational interaction be- tween molecules adsorbed on a metal surface: The dipoledipole interaction. Phys. Rev. B 1981, 24, 6954-6970.

50 Tyrode, E.; Corkery, R. Charging of carboxylic acid monolayers with monovalent ions at low ionic strengths: Molecular insight revealed by vibrational sum frequency spectroscopy. J. Phys. Chem. C 2018, 122, 28775-28786.

51 Sthoer, A.; Tyrode, E. Interactions of $\mathrm{Na}^{+}$cations with a highly chharged fatty acid Langmuir monolayer: Molecular description of the phase transition. J. Phys. Chem. C 2019, 123, 23037-23048.

52 Fainerman, V. B.; Vollhardt, D.; Johann, R. Arachidic acid monolayers at high $\mathrm{pH}$ of the aqueous subphase: Studies of counterion binding. Langmuir 2000, 16, 7731-7736.

53 Johann, R.; Vollhardt, D. Texture features of long-chain fatty acid monolayers at high $\mathrm{pH}$ of the aqueous subphase. Mat. Sci. Eng. C 1999, 8-9, 35-42.

54 ad C. B. Casper, E. M. A.; Allen, H. C. Effect of cation enrichment on dipalmitoylphosphatidylcholine (DPPC) monolayers at the air-water interface. J. Colloid Interf. Sci. 2016, 478, 353-364.

55 Javanainen, M.; Hua, W.; Tichacek, O.; Delcroix, P.; Cwiklik, L.; Allen, H. C. Structural effects of cation binding to DPPC monolayers. Langmuir 2020, 36, 1525815269 .

56 Adams, E. M.; Verreault, D.; Jayarathne, T.; Cochran, R. E.; Stone, E. A.; Allen, H. C. Surface organization of a DPPC monolayer on concentrated $\mathrm{SrCl}_{2}$ and $\mathrm{ZnCl}_{2}$ solutions. Phys. Chem. Chem. Phys. 2016, 18, $32345-32357$.

\section{Table of Contents Graphic}

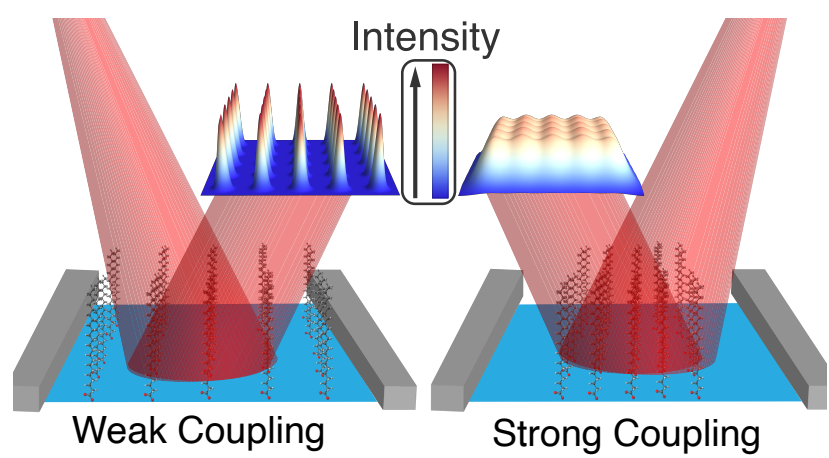

